\title{
Quantitative characterization on the microscopic pore heterogeneity of tight oil sandstone reservoir by considering both the resolution and representativeness
}

\author{
Shuheng Du ${ }^{\mathrm{a}, \mathrm{b}, \mathrm{c}, *}$, Shan Pang ${ }^{\mathrm{a}, \mathrm{c}}$, Yongmin Shi ${ }^{\mathrm{c}}$ \\ ${ }^{a}$ School of Earth and Space Science, Peking University, Beijing, 100871, China \\ ${ }^{\mathrm{b}}$ Institute of Mechanics, Chinese Academy of Sciences, Beijing, 100190, China \\ ' Oil and Gas Institute, Peking University, Beijing, 100871, China
}

\section{A R T I C L E I N F O}

\section{Keywords:}

"Umbrella deconstruction" method

Microscopic heterogeneity

Tight oil reservoir

\begin{abstract}
A B S T R A C T
This study aimed to raise the new experiment method named "umbrella deconstruction" to reveal the microscopic pore heterogeneity of tight oil sandstone reservoir by considering both the resolution and representativeness. Based on the FE-SEM and image processing technology, parameters like porosity, the ratio of the maximum to the minimum of pore orientation frequency, the content of cement, the ratio of the maximum to the minimum of cement orientation frequency and the fractal dimension of reservoir space could be applied into the microscopic heterogeneity characterization. The final imaging observation scale could reach approximately $20 \mathrm{~mm}$ (which is 10 times CT observation scale), the precision can reach $10 \mathrm{~nm}$ (almost equal to the CT observation resolution). This method would help to the research work in the microscopic heterogeneity characterization of unconventional reservoir.
\end{abstract}

\section{Introduction}

Rock slicing is the most direct and effective way to study the development of minerals and pores in any type of reservoir. A series of major breakthroughs in reservoir geology, such as identification of pore and throat, and discovery of nano pores in unconventional hydrocarbon reservoir, all benefit from the observation and testing technology of rock slices (Zhao et al., 2018; Kareem et al., 2017; Mayo et al., 2015).

For the continental tight sandstone reservoirs in China, due to the frequent migration of the channels, strong diagenesis and transformation of the minerals, there is significant microscopic heterogeneity in minerals development and pore throat distribution in the reservoir. In recent years, the research ideas on microscopic heterogeneity of reservoir are mainly focused on micro and nano CT scanning and "focused ion beam-scanning electron microscopy(FIB-SEM)". Through the threedimensional reconstruction of the CT data, the anisotropy and microscopic heterogeneity of the reservoir is studied (Tisato et al., 2016; Saxena et al., 2017; Rees et al., 2011). Therefore, research scholars mostly aimed to figure out how to improve the accuracy of observation but actually ignored the existing technical conditions which is the observation accuracy greater, the required sample size must be smaller, then the heterogeneity of the sample would be weaker (BarakaLokmane et al., 2009; Liu et al., 2017; Tiwari et al., 2013). It is deviated from the original research object which is the heterogeneity characterization. So the study of the microscopic heterogeneity of unconventional hydrocarbon reservoirs under the premise of both resolution and representativeness is worth exploring.

\section{Principle}

Based on the analysis of the former studies, the authors put forward the "umbrella deconstruction" method, tried to find a method that can take account of both the resolution and representativeness to extract the information from rock samples more directly so that we could study the heterogeneity of reservoir more flexible. We try to develop a new method for characterizing the pore \& throat anisotropy of tight oil reservoirs in large sight effectively which could give consideration to both the resolution and scale (London et al., 2014; Baniak et al., 2013; Alyafei et al., 2016).

In Fig. 1 and Fig. 2, first, we drilled the standard core sample of the unconventional hydrocarbon reservoir. Second, we draw 8 remarkable lines every $22.5^{\circ}$ in the overlook surface of the sample. Third, we cut the thin sections along the 8 lines. Fourth, we use the FE-SEM instrument to characterize the reservoir in 8 thin sections and get a large amount of images in small view. Fifth, we spliced all the images in small view into 8 images in large view. Finally, we could carry out the

\footnotetext{
* Corresponding author. No.5 Yiheyuan Road, Haidian District, Beijing, China.

E-mail address: dushuheng@pku.edu.cn (S. Du).
} 